Nork performed under the avipises of the l'S. Thenttment of Fenery by Insence Jiermore Vetionsl Isbors. tan) under contract number \$-7405-EYG-48.

This document mes prepared as an atcount of nork sponsored by an akchey of the linited Stules Goretnoment. thilker the linied Stales Government wor the Cininessily of California nor any of their tmplọeses, makes any narranty.

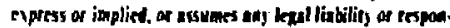
sibility for the acrufacy. completeness, of usfulness of any information, apparatus, product, of process disstosedh or Ippresents that its dse nould not intringe privately onsed tiphts. Reference herein to eny speeifis cemnerrial prod.

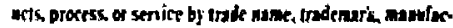
twrer, or otherwise. does nol necessurily consitute or inply its endorsement. recommendatiom or fouriag bs the United Siates Gou ernment or the t'ninersity of Californis. The

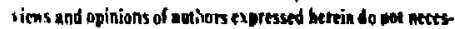
sarily stale or refhet those ov (he Uaited Stals Goienmest or the l'niversity of Califonihn, and stail on in aed for adiertising or prodect endorsement parposer. 


\title{
Parameters and Error of a Theoretical Model
}

\author{
P. Möller \\ Lawrence Livermore National Laboratory, Livermore, CA 94550
}

J. R. Nix

Theoretical Division, Los Alamos National Laboratory, Los Alamos, NM 87545

August 27, 1986

\begin{abstract}
We propose a definition for the error of a theoretical model of the type whose parameters are determined from adjustment to experimental dats. By applying a standard atatistical method, the marimum-litelihood method, we derive expressions for both the parometers of the theoretical model and its ersor. We investigste the derived equations by wolving them for simulated experimental and theoretical quantities generated by use of tandom number generators.
\end{abstract}

We discuss some aspects of how to estimate the parameters and the error of a theoretical model. We assume that the parameters and the error are estimated by adjustments and comparisons to experimental data. Our interest in this study was motivated by our desire to devise a method for defining and determining the error of a theoretical model for nuclear masses. The notation will therefore, for concreteness and simplicity, be tailored to the example of a theoretical nuclear mass model.

In some earlier studies, the error $\sigma_{\mathrm{th}}$ of a theoretical mass model was somewhat atbritrarily defined as

$$
\sigma_{\text {th }}=\left[\frac{1}{n} \sum_{i=1}^{n}\left(M_{\text {uxp }}^{i}-M_{\mathrm{th}}^{i}\right)^{2}\right]^{\frac{1}{2}}
$$

Here $M_{\operatorname{cop}}^{i}$ is the measured mass for a particular value of the proton number $Z$ and neutron number $N$, and $M_{\mathrm{tb}}^{\mathrm{i}}$ is the corresponding calculated quantity. There are $n$ such measurements for different $N$ and $Z$. The theoretical model for $M_{\mathrm{th}}^{i}$ also depends on $m$ adjustable parameters $p_{1}, p_{2}, \ldots, p_{m}$. The definition (1) is reasonable when all

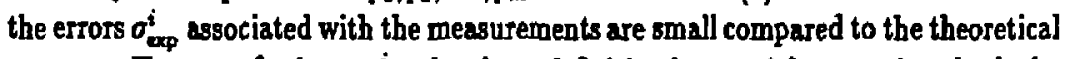
errot $\sigma_{\mathrm{th}}$. However, for large $\sigma_{\mathrm{ep}}^{i}$ the above definition is unsatisfactory, since both the theoretical and experimental errors contribute to $\sigma_{\mathrm{kb}}$. The definition (1) will therefore always overestimate the theoretical error. We must therefore use an approach that decouples the theoretical and experimental errors from one another.

First we specify our assumptions, defining precisely what we mean by the error of a theory. Our model $M_{\mathrm{th}}$ for nuclear masses is a sum of twenty or so terms. There are a few large terms, but many more terms that give fairly small contributions to the expression. This structure of the model has arisen because one has tried to develop the 
model over the years by adding new terms that describe previously neglected effects. Origina.'y the model expression consisted of only a few large terms describing the most important effects. There are numerous effects of a smaller magnitude that have later been included as additional terms in the model. It is reasonable to wsume that there are an even larger number of terms that account for even smaller effects that have been left out of the model. Thus the model has an error associated with these missing terms. We now want to characterise this error and estimate its magnitude. Since we assume that the error is due to a large number of missing terms that are small and fuctuating, we treat the problem with statistical methods.

A general method for determining unknown parameters of a known statistical distribution if one has observed events distributed according to the distribution is the maximum likelihood method. For an in-depth discussion of this method, notation and fundamental concepts of statistics we refer to the books by Cramer and von Mises $[1,2]$. According to this method one estimates the parameters so that the events that have been observed have the maximum likelihood of being observed. Suppose that $\xi^{i}$ is distributed according to $f_{\xi^{i}}(x, \theta)$, with un'nown. If we have observed $\xi^{1}=x^{1}, \xi^{2}=x^{2}, \ldots, \xi^{n}=x^{n}$, we can determine $\theta$ so that our observations have maximum likelihood. This is done by maximizing the likelihood function $L(\theta)$, where

$$
L(\theta)=f_{\xi^{1}}\left(x^{1}, \theta\right) f_{\xi^{2}}\left(x^{2}, \theta\right) \ldots f_{\xi^{n}}\left(x^{n}, \theta\right)
$$

To find the maximum of the likelihood function $L(\theta)$ is equivalent to maximizing the logarithm $\ln [L(\theta)]$, or to solving

$$
\frac{\partial[\ln L(\theta)]}{\partial \theta}=0
$$

We denote the solution of eq. (3) by $\theta^{*}\left(x^{1}, x^{2}, \ldots, x^{n}\right)$. By solving eq. (3) for several sets of observations $x_{i}^{1}, x_{i}^{2}, \ldots, x_{i}^{n}$ of the set of random variables $\xi^{1}, \xi^{2}, \ldots, \xi^{n}$, we obtain several eatimates $\theta_{i}^{*}$ of the parameter $\theta$ of the distribution functions $f_{\xi^{i}}(x, \theta)$. Thus $\theta^{\circ}\left(x^{1}, x^{2}, \ldots, x^{n}\right)$ is an observation of a random variable $\theta^{\circ}\left(\xi^{j}, \xi^{2}, \ldots, \xi^{n}\right)$ with a distribution function $90^{*}$. Since the distribution function of the multidimensional random variable $\left(\xi^{1}, \xi^{2}, \ldots, \xi^{n}\right)$ is known (it is actually the product in the right member of eq. (2)), the distribution function of $\theta^{*}\left(\xi^{1}, \xi^{2}, \ldots, \xi^{n}\right)$ can be determined, at least in principle. This is of fundamental importance. It is desirable that its distribution function $g_{0}$. has certain properties. This is an important field of mathematical statistics, and we refer to the books by Cramér and von Mises $[1,2]$ for a discussion of these questions. Here we just mention that the maximum-likelihood (ML) estimate is consistent, that is

$$
\lim _{n \rightarrow \infty} \mathbf{E}\left(\theta^{*}\right)=\theta, \quad \lim _{n \rightarrow \infty} \mathbf{D}^{2}\left(\theta^{*}\right)=0
$$

where $\mathbf{E}\left(\theta^{\circ}\right)$ denotes the mean and $\mathbf{D}^{\mathbf{2}}\left(\theta^{\circ}\right)$ the variance of the distribution function of $\theta^{*}$. One can also show that if an efficient estimate $\theta^{*}$ of exists, then the likelihood equation will have a unique solution equal to $\theta^{*}$. An efficient estimate is an estimate 
that is unbiased and has minimum variance. The estimate is said to be unbiased if

$$
\mathbf{E}\left(\theta^{\circ}\right)=\theta
$$

for all $n$. However, many ML estimates have bias.

For the case in which the ML estimate is biased, one may ask if an unbiased estimate is a better one. However, even if the ML eotimate is biased, it is consistent and therefore the parameter estimates are arbritralily close to the true values for sufficiently large $n$, provided that the number of parameters does not increase as $n$ increases. One may therefore agree with von Mises (ref. [2], p. 560) that if for the actual purpose of an investigation the difference between an ML estimate and an uabiased estimate is of importance, lasger sample should have been chosen.

For the specific case of nuclear mass formulae, it is not possible to substantially increase the number of data points. Thus one should only consider models in which relatively few parameters are adjusted to the data. The adjustment of up to about 500 parameters to a data set of about 1500 experimental masses leads to very unreliable estimates of the parameters from a statistical point of view (we also have serious objections to such models from a physical point of view), and therelore to poor properties for extrapolation to new regions of nrclei. If one persists in studying models with a iarge number of adjustable parameters compared to the available data one should study the effect of bias in the estimate below in eq. (10) for $\sigma_{\mathrm{hh}}{ }^{2}$, since for a large number $m$ of adjustable parameters we expect that approximately (cf. ref. [2], p. 555)

$$
\mathbf{E}\left(\theta^{\prime}\right) \simeq \frac{n-m}{n} \sigma_{\mathrm{th}}{ }^{*}
$$

The unknown parameters of our theory can be determined by use of the ML method only if they are parameters of the distributions involved. In particular, they can not be parameters of the prescription to generate the variables of the distribution function. Thus, we must formulate the problem so that $M_{i k}$ becomes a parameter of the distribution. This can be done in the following way.

We write $u_{\mathrm{tr}}^{i}=M_{\mathrm{th}}^{i}+e_{\mathrm{th}}^{i}$, where $e_{\mathrm{th}}^{i} \in \mathrm{N}\left(0, \sigma_{\mathrm{th}}{ }^{2}\right)$ is an error term and $M_{\mathrm{th}}^{i}$ is our model for the nuclear mass. We are now in a position to give a precise definition of the error of the theory, which we designate by the quantity $\sigma_{\text {th }}$. The variable $u_{\mathrm{tr}}^{i}$ is the true mass of the nucleus $i$, where $i$ stands for a particular value of the proton number $Z$ and and the neutron number $N$. However, $u_{\mathrm{tr}}^{i}$ is also a random variable, by construction.

The following somewhat drastic example clarifies this duality. Suppose that when God originally created the Universe and fixed each nuclear mass $i$, He used our expression for $M_{\text {th }}^{i}$, with some parameters He fixed and which it is now up to us to estimate. To the number He obtained by calculating $M_{\mathrm{th}}^{i}$ He added a random number $e_{\mathrm{th}}^{i}$ which He obtained by throwing dice such that $e_{\mathrm{ch}}^{i} \in \mathrm{N}\left(0, o_{\mathrm{th}}{ }^{2}\right)$. The random variable $e_{\mathrm{th}}^{i}$ represents the unknown terms that are missing from the theory and $\sigma_{\text {th }}$ is by definition the error of the theory.

We note that in practice there are correlations in the theoretical error, in the sense that the error for calculated masses is of similar magnitude if the $Z$ and $N$ values do 
not differ too much. However, this is of no consequence for the considerations below, since we consider a large number of masses throughout the periodic system. Were one to consider the differences between theoretical masses, whose $Z$ and $N$ values differ by only one or two units, correlations would be important and would reault in etrors in the differences that are much smaller than $\sqrt{2} \sigma_{\text {th }}$, which would be expected in the absence of correlations.

The experimental quantities are not observations of precisely $u_{\mathrm{tr}}^{i}$, but are instead observations of the random variable $v_{\text {exp }}^{i}=u_{\mathrm{tr}}^{i}+c_{\mathrm{exp}}^{i}=M_{\mathrm{th}}^{i}+e_{\mathrm{th}}^{i}+e_{\mathrm{exp}}^{i}$, because there are random errors associated with the measuring process. We assume that $e_{\operatorname{axp}}^{i} \in N\left(0, \sigma_{\operatorname{axp}}^{i}\right)$. The $\sigma_{\operatorname{axp}}^{i}$ are different for different $i$ because the measuring device is different for each $i$, but they are all known. From the above conditions we find that $v_{\operatorname{cop}}^{i} \in \mathrm{N}\left(M_{\mathrm{th}}^{i}, \sigma_{\operatorname{sxp}}^{i}+\sigma_{t h}{ }^{2}\right)$. Thus, for the distribuiton function $f_{v^{i}}$ we have

$$
f_{v i}\left(x, M_{\mathrm{th}}^{i}, \sigma_{\mathrm{opp}}^{i^{2}}+\sigma_{\mathrm{th}}{ }^{2}\right)=\frac{1}{\sqrt{2 \pi}} \frac{1}{\sqrt{\sigma_{\mathrm{app}}^{i}+\sigma_{\mathrm{th}}{ }^{2}}} e^{-\frac{\left(x-M_{\mathrm{th}}^{i}\right)^{2}}{2\left(\sigma_{\mathrm{exp}}^{i}{ }^{2}+\sigma_{\mathrm{th}}{ }^{2}\right)}}
$$

The values $M_{\exp }^{i}$ constitute observations of $v_{\exp }^{i}$. We can now estimate both the parameters $p_{\nu}$ of $M_{\mathrm{th}}^{i}$ and the quantity $\sigma_{\mathrm{th}}$ by maximizing the likelihood function $L$. In our case

$$
L\left(p_{1}, p_{2}, \ldots, p_{m}, \sigma_{t h}^{2}\right)=\int_{v^{1}}\left(x, M_{t h}^{1}, \sigma_{\exp }^{1}{ }^{2}+\sigma_{t h}^{2}\right) \ldots f_{v n}\left(x, M_{t h}^{n}, \sigma_{\exp }^{n}{ }^{2}+\sigma_{t h}^{2}\right)
$$

Maximizing $L$ is equivalent to solving the $m+1$ equations

$$
\sum_{i=1}^{n} \frac{\left(M_{e x p}^{i}-M_{\mathrm{th}}^{i}\right)}{\sigma_{\exp }^{i}+\sigma_{\mathrm{th}}{ }^{2}} \frac{\partial M_{\mathrm{th}}^{i}}{\partial p_{\nu}}=0, \quad \nu=1,2, \ldots, m
$$

and

$$
\sum_{i=1}^{n} \frac{\left(M_{\mathrm{exp}}^{i}-M_{\mathrm{th}}^{i}\right)^{2}-\left(\sigma_{\mathrm{exp}}^{i}+\sigma_{\mathrm{th}}{ }^{2 *}\right)}{\left(\sigma_{\mathrm{exp}}^{i}+\sigma_{\mathrm{th}}{ }^{2+}\right)^{2}}=0
$$

Here the notation $\sigma_{\mathrm{th}}{ }^{2 *}$ means that by solving eq. $(10)$ we obtain the estimate $\sigma_{\mathrm{th}}{ }^{2 *}$ of the true $\sigma_{t h}{ }^{2}$. The above equations are equivalent to mirımizing $S$ with respect to $p_{v}$,where

$$
S=\sum_{i=1}^{n} \frac{\left(M_{\mathrm{exp}}^{i}-M_{\mathrm{tb}}^{i}\right)^{2}}{\sigma_{\mathrm{epp}}^{i}+\sigma_{\mathrm{th}}^{2+}}
$$

and solving

$$
\sum_{i=1}^{n} \frac{\left(M_{\exp }^{i}-M_{\mathrm{th}}^{i}\right)^{2}-\left(\sigma_{\exp }^{i}+\sigma_{\mathrm{th}}{ }^{2 *}\right)}{\left(\sigma_{\operatorname{cop}}{ }^{2}+\sigma_{\mathrm{th}}{ }^{2 *}\right)^{3}}=0
$$

Thus we are led to one additional equation relative to the usual set of least-square equations that arise when model parameters are estimated by adjustments to experimental data under the assumption of a perfect theory, that is $\sigma_{\mathrm{th}}{ }^{2}=0$. Equations 
$(9,10)$, or alternatively, eqs. $(11,12)$ constitute a system of $m+1$ equations that are to be solved together.

It is instructive to rewrite eq. (12) as

$$
\sigma_{\mathrm{th}}^{2}=\frac{1}{\sum_{i=1}^{n} w_{i}} \sum_{i=1}^{n} w_{i}\left[\left(M_{\exp }^{i}-M_{\mathrm{th}}^{i}\right)^{2}-\sigma_{\exp }^{i}{ }^{2}\right]
$$

where

$$
w_{i}=\frac{1}{\left(\sigma_{\exp }^{i}+\sigma_{\mathrm{th}^{2 i}}\right)^{2}}
$$

and

$$
k=2
$$

Written in this way the unknown $\sigma_{t h}{ }^{2 *}$ enters on both sides of the equal sign in eq. (13), but the equation is suitable for an iterative determination of $\sigma_{\mathrm{th}}{ }^{{ }^{*}}$. We have found that the convergence is extremely rapid.

Another benefit of rewriting eq. (10) in the form givtn by eq. (13) is that one may ask if $k=2$ is the only possibility, or if other values of $k$ are possible. We shall not discuss this question in depth but conclude this report with a few comments on this matter. First, we observe that if all ${\sigma_{\text {oxp }}^{i}}^{2}$ are equal, then all values of $k$ yield the same equation. However, the more interesting case is how $k$ should be chosen if the $\sigma_{\exp }^{i}$ have different values. According to our discussion above, one can prove that the ML estimate has several desirable properties. About other possible $k$ values we here prove nothing, but to gain some insight we compare the ML estimate corresponding to $k=2$ with estimates obtained with $k=0,1,3$ and 4 by simulating the random varjables $e_{\text {th }}^{i}$ and $e_{\exp }^{i}$ We do 4 simulations, each with $n=200$, the results of which are given in tables 1 through 4. In each simulation we generate one set of 200 values for $e_{\text {exp. In }}^{i}$ each case we also generate 2000 sets of 200 values for $e_{\mathrm{th}}^{i}$, with $\sigma_{\mathrm{th}}=1.0$ in all cases. Equation (12) is solved for each of the 2000 sets. In this way we estimate the mean and variance of $\sigma_{\mathrm{tb}}{ }^{{ }^{\circ}}$, which is distributed according to $g_{0 .}$. In table 1 the $\sigma_{\exp }^{i}$ are distributed evenly in the interval 0 to 10 , and in table 2 they are distributed evenly in the interval 0 to 3 . In tables 3 and 4 the $\sigma_{\text {app }}^{i}$ are distributed approximately like those for the 200 most recently discovered experimental masses. The difference between table 3 and table 4 is just a different seed for the generation of the experimental quantities. From the results of the simulations we see that the means are usually larger than 1.0 , an indication that the satimates have bias. We also see that except for table 3 , $k=2$ yields the smallest estimate for the variance $D^{2}\left(\theta^{n}\right)$. That $k=1$ yields the smallest estimate in table 3 probably indicates that we need to do more simulations with different seeds for the distribution of $\sigma_{\text {exp }}^{i}$ to reliably estimate $D^{2}\left(\theta^{*}\right)$. The results of the simulations illustrate the good qualities of the ML estimate, in accordance with our theoretical considerations. 
Table 1

\begin{tabular}{lrrrrr}
\multicolumn{5}{c}{ Results for $0 \leq \sigma_{\text {ap }}^{i} \leq 10$} \\
\hline$k$ & 0 & 1 & 2 & 3 & 4 \\
$\mathrm{E}\left(\theta^{\circ}\right)$ & --- & 0.9884 & 1.0177 & 1.0167 & 1.0194 \\
$\mathrm{D}^{2}\left(\theta^{\circ}\right)$ & -- & 0.02511 & 0.02307 & 0.02583 & 0.02825 \\
$\mathrm{D}\left(\theta^{\circ}\right)$ & -- & 0.15848 & 0.15187 & 0.16071 & 0.16807 \\
\hline
\end{tabular}

Table 2

\begin{tabular}{lrrrrr}
\multicolumn{5}{c}{ Results for $0 \leq \sigma_{\text {exp }}^{i} \leq 3$} \\
\hline$k$ & 0 & 1 & 2 & 3 & 4 \\
$\mathrm{E}\left(\theta^{*}\right)$ & 1.0686 & 1.0060 & 1.0098 & 1.0207 & 1.0278 \\
$\mathrm{D}^{2}\left(\theta^{*}\right)$ & 0.01586 & 0.00808 & 0.00769 & 0.00868 & 0.00986 \\
$\mathrm{D}\left(\theta^{*}\right)$ & 0.12594 & 0.08991 & 0.08767 & 0.09316 & 0.09929 \\
\hline
\end{tabular}

Table 3

\begin{tabular}{lrrrrr}
\multicolumn{5}{c}{ Results for $\sigma_{\exp }^{i}$ distributed as in experiment } \\
\hline$k$ & 0 & 1 & 2 & 3 & 4 \\
$\mathrm{E}\left(\theta^{*}\right)$ & 0.9361 & 0.9951 & 1.0065 & 1.0094 & 1.0103 \\
$\mathrm{D}^{2}\left(\theta^{*}\right)$ & 0.00318 & 0.00260 & 0.00263 & 0.00266 & 0.00269 \\
$\mathrm{D}\left(\theta^{\circ}\right)$ & 0.05637 & 0.05103 & 0.05127 & 0.05161 & 0.05188 \\
\hline
\end{tabular}

Table 4

Results for $\sigma_{\text {exp }}^{i}$ distributed as in experiment

\begin{tabular}{lrrrrr}
\hline$k$ & 0 & 1 & 2 & 3 & 4 \\
$\mathrm{E}\left(\theta^{*}\right)$ & 1.0617 & 1.0275 & 1.0139 & 1.0106 & 1.0097 \\
$D^{2}\left(\theta^{*}\right)$ & 0.00358 & 0.00276 & 0.00271 & 0.00272 & 0.00271 \\
$\mathrm{D}\left(\theta^{*}\right)$ & 0.05986 & 0.05256 & 0.05202 & 0.05215 & 0.05204 \\
\hline
\end{tabular}

\section{References}

1. H. Cramér, Mathematical Metods of Statistics, Princton University Press, 1946.

2. R. von Mises, Mathematical Theory of Probability and Statistics, Academic Press, New York, 1964. 